\title{
FIRST COUNTABLE, COUNTABLY COMPACT, NONCOMPACT SPACES
}

\author{
PETER NYIKOS
}

The main problem in this article is one of my all-time favorites. To drum up interest in it, I announced at the 1986 Prague Topological Symposium (Toposym) that I was offering a US $\$ 500$ prize for a solution during the following ten years [9]. There was essentially no progress on the problem all during those ten years, and so at the 1996 Toposym I raised the award to US\$1000 during the following ten years. Those ten years have almost passed with no progress on the problem at all to the best of my knowledge, and I am hereby removing all time limits on the $\$ 1000$ award and am contemplating raising it. Here is the problem that is the focus of all this largesse:

Problem 1. Does ZFC imply the existence of a separable, first countable, countably compact, noncompact Hausdorff $\left(T_{2}\right)$ space?

A mild put-down of general topology one hears from time to time is that there are too many adjectives in a typical problem or theorem. For me, however, one of the charms of general topology is that there are so many theorems and problems one can understand with no more than a typical undergraduate textbook in general topology as a resource. The adjectives used here definitely fall under that heading; the concepts are like second nature to many of us, and I have little mental pictures that I associate to each one to help keep arguments straight.

I will soon cut down on the number of adjectives in the alternative wording below, but the ones in the original wording are all implicitly there. The usual topology on $\omega_{1}$ satisfies everything except separability. The Novak-Teresaka space described in Vaughan's article [15] satisfies everything except first countability. If one refines the cofinite topology on $\omega_{1}$ by making initial segments open, then the resulting space satisfies everything except $T_{2}$ and is $T_{1}$. The remaining two properties are obviously necessary also to have an open problem. Also, the question mentions ZFC because there is a multitude of consistent examples of spaces as in Problem 1; see Sections 1 and 2. In fact, Problem 1 is one of a small but growing number of topological problems for which a negative answer is known to entail $\left(2^{\omega}=\right) \mathfrak{c} \geq \aleph_{3}$, yet $\mathfrak{c}=\aleph_{3}$ has not been ruled out. See Section 4 .

2000 Mathematics Subject Classification. 54A25, 54A35, 54D20, 54D45, 54G20.

Key words and phrases. countably compact, first countable, separable, $\omega$-bounded, locally compact, locally countable, good, splendid, Chang Conjecture variant, fine, amenable, Kurepa family. 
For reasons having nothing to do with the aforementioned put-down, it is convenient to introduce the following concepts:

Definition. A space $X$ is $\omega$-bounded if every countable subset has compact closure, and strongly $\omega$-bounded if every $\sigma$-compact subset has compact closure.

Also, after this paragraph, space will mean $T_{2}$-space. In fact, it could almost as easily mean $T_{3}$-space because of the well-known fact that $T_{2}$ implies $T_{3}$ for first countable, countably compact spaces $[1,2]$. (However, this doesn't work with "locally countable" in place of "first countable," as shown by an example of mine, mentioned in Vaughan's article [15] and done in detail in [14].) I could also have gone quite far in the opposite direction: an easy proof by contrapositive shows that every first countable space in which convergent sequences have unique limits is $T_{2}$. With this convention, we give the negative version of Problem 1 thus:

Problem $\mathbf{1}^{\prime}$. Is it consistent that every first countable, countably compact space is $\omega$-bounded?

Unlike with Problem 1, we have no consistency results either way for the following problem:

? 1002 Problem 2. Is there a first countable, $\omega$-bounded space that is not strongly $\omega$ bounded?

The following ZFC example shows that first countability cannot be dropped from this problem.

Example. Let $p$ be a weak P-point in $\omega^{*}=\beta \omega \backslash \omega$ that is not a P-point. Then $\omega^{*} \backslash\{p\}$ is locally compact, $\omega$-bounded, and not strongly $\omega$-bounded.

\section{Consistent good examples for Problem 1}

The best-known consistent examples for Problem 1 are the separable uncountable good spaces that are also discussed in the articles by Juhász [5] and Vaughan [15].

Definition. A space is good if it is countably compact, locally countable, and $T_{3}$. A space is splendid if it is good and $\omega$-bounded.

Clearly, a good space is noncompact iff it is uncountable, and so a good space is splendid iff every countable subset has countable closure. It follows that a ZFC example of good space that is not splendid would solve Problem 1. We do not know whether the added generality in Problem 1 is necessary - in other words, part (a) of the following problem is unsolved; so is part (b):

? 1003-1004 Problem 3. Is it consistent that there is a countably compact first countable space that is not $\omega$-bounded and yet (a) every good space is splendid, or (b) every locally compact, countably compact first countable space is $\omega$-bounded?

Clearly, every good space is locally compact. In [11] I gave a general construction of separable good spaces that are not splendid, referring to such spaces as 
Ostaszewski-van Douwen spaces. This is the Ostaszewski construction by induction explained in detail in Vaughan's article [15], with one modification stemming from the fact that we do not care whether the spaces are $r$-compact for some ultrafilter $r$.

At the $\alpha$ th step $(\alpha<\mathfrak{c})$ we have a locally compact, locally countable noncompact space $\left(\alpha, \mathcal{T}_{\alpha}\right)$ with $\omega$ as a dense set of isolated points. Having earlier listed all sequences $s$ on $\mathfrak{c}$ in a $\mathfrak{c}$-sequence, we take the first sequence $s_{\beta}$ without a cluster point; if there is none, then $\left(\alpha, \mathcal{T}_{\alpha}\right)$ is a good space. Otherwise, the crucial question is whether $\left(\alpha, \mathcal{T}_{\alpha}\right)$ is a $\mathrm{wD}$ space:

Definition. A space $X$ is $w D$ if for every infinite closed discrete subspace $D$ there is an infinite $E \subset D$ for which there is a discrete family of open sets $U_{e}$ such that $U_{e} \cap E=\{e\}$ for all $e \in E$.

If $\left(\alpha, \mathcal{T}_{\alpha}\right)$ is a wD space, then we select an infinite $E$ as above, and choose $U_{e}$ to be a compact (hence countable) open neighborhood of $e$, cutting down $E$ if necessary so that when we make $\bigcup_{e \in E} U_{e} \cup\{\alpha\}$ the one-point compactification of $\bigcup_{e \in E} U_{e}$ and add $\alpha$ to $\left(\alpha, \mathcal{T}_{\alpha}\right)$, the resulting space $\left(\alpha+1, \mathcal{T}_{\alpha+1}\right)$ remains noncompact. Once $\alpha \geq \omega_{1}$ this reason for cutting down $E$ becomes obsolete (although we may have other reasons for cutting it down, see below) because $\left(\alpha, \mathcal{T}_{\alpha}\right)$ is automatically noncompact, being uncountable and locally countable. If we can define $\left(\alpha, \mathcal{T}_{\alpha}\right)$ for all $\alpha<\mathfrak{c}$ then we have a good space at the end, because every sequence has been given a cluster point. In the article by Vaughan [15], various models are given where the construction ends in a good space, either by continuing all the way to $\mathfrak{c}$ or stopping earlier with all sequences having cluster points. In particular, in any model where $\mathfrak{b}=\mathfrak{c}$, the construction can (if so desired) continue to stage $\mathfrak{c}[3$, Theorem 13.4]. In some models we have no choice, such as models where $\mathfrak{p}=\mathfrak{c}$; see [4] where it is shown that every $T_{3}$ separable, countably compact space of Lindelöf number $<\mathfrak{p}$ is compact. In others we do have a choice (such as models where $\mathfrak{p}=\omega_{1}$, see below).

If at some point $\left(\alpha, \mathcal{T}_{\alpha}\right)$ is not a $\mathrm{wD}$ space, and we do not yet have a good space, then we have to scrap it and try again. It cannot be extended to a space for Problem 1 because of the following fact:

Theorem 1.1 ([11, Theorem 1.3]). Every subspace of a first countable, countably compact space is a $w D$ space.

Moreover, in a certain sense, we have to modify various choices of $U_{e}$ and/or the set to which $E$ is cut down and/or the order in which $s_{\beta}$ is listed: as explained in [11], every separable good noncompact space admits a construction such as we have gone through just now.

This is not to say that there might not be other ways of constructing the same space. In [10] there is a construction which begins with a splendid space of cardinality $\mathfrak{c}$ and repeatedly tears chunks from it, attaching the chunks to a copy of $\omega$ which will be dense in the intermediate spaces. As explained in [11], however, the same obstacle of an intermediate non-wD space might be encountered before we have a countably compact space on our hands. There is the added incovenience 
that in some models obtained using enormously large cardinals, all splendid spaces are of cardinality less than $\mathfrak{c}$; see Section 3 .

A different alternative construction is in models of $\mathfrak{p}=\omega_{1}$, which is equivalent [3] to $\mathfrak{t}=\omega_{1}$, i.e., there is a decreasing mod-finite $\omega_{1}$-tower on $\omega$. This is a family $\left\{A_{\alpha}: \alpha \in \omega_{1}\right\}$ of infinite subsets of $\omega$ such that $A_{\alpha} \subset^{*} A_{\beta}$ whenever $\alpha>\beta$, and such that no infinite subset $\subset^{*} A_{\alpha}$ for all $\alpha<\omega_{1}$. Given such a family, one constructs a Franklin-Rajagopalan (FR) space as explained in Vaughan's article [15], a countably compact space with $\omega$ as a dense set of isolated points and set of non-isolated points homeomorphic to $\omega_{1}$. Like all FR-spaces it is locally compact, and so it is a good space. As usual, it is defined "all in one go" in [15] but it could also be constructed by the Ostaszewski technique and provides us with an example where the construction ends before stage $\mathfrak{c}$ in any model of $\mathfrak{t}=\omega_{1}<\mathfrak{c}$.

Yet another approach to constructing good spaces is in the last section of [11]: construct good spaces in ground models or intermediate models with a view to them being preserved in forcing extensions. Unfortunately, I know of no progress here since [11] was written; the results sketched there have not even been published yet.

\section{Other consistent constructions for Problem 1}

In this section we summarize constructions of separable, countably compact, first countable, noncompact spaces that are not good. None to date has given us examples in any model where there are good examples for Problem 1.

Example ([11, p.139]). Modify the Ostaszewski construction to begin with $2^{\omega}(=$ the Cantor set) $\times \omega$, to serve as a dense subspace for the rest of the inductive construction. At stage $\alpha$ we could take advantage of the fact that the union of the $U_{e}$-analogues is homeomorphic to $2^{\omega} \times \omega$ and to compactify it by identifying it with an open subspace of a copy $\mathbb{C}_{\alpha}$ of the Cantor set, the rest of $\mathbb{C}_{\alpha}$ being disjoint from the space we have constructed thus far. The special case where there is only point in the rest of $\mathbb{C}_{\alpha}$ is especially close to the Ostaszewski construction, allowing for $[\omega, \alpha)$ to be the rest of the space at stage $\alpha$.

Example ([8, Example 3.11]). Begin with an open ball in $\mathbb{R}^{n}(n \geq 2)$ and recursively add copies of $[0,1)$ in a way that makes the spaces we build into $n$-manifolds with the original open ball as a dense subspace.

$\mathrm{CH}$ and a few forcing models are enough to give us countably compact noncompact manifolds (hence locally compact in addition to being first countable), but this seems too restrictive a method of constructing spaces for Problem 1 . The following problem from [11], for example, is still unsolved.

? 1005 Problem 4. Is it consistent for there to be a countably compact manifold of weight $>\omega_{1}$ ?

Lacking a Yes answer, we are stymied in all models of $\mathfrak{p}>\omega_{1}$, while $\mathfrak{p}=\omega_{1}$ is already enough to give us a good FR-space (see above). Section 6 of [11] details 
the main hurdles to any solution of Problem 4. The key problem is that $\mathrm{wD}$ is no longer good enough to continue the construction if it is not yet countably compact at stage $\alpha$; one needs for there to be a subsequence of $s_{\alpha}$ contained in a closed copy of the closed ball minus a single point, and there are ZFC examples of weight $\omega_{1}$ where there is no such subsequence.

Example ([11, Section 5]). A countably compact, first countable linearly ordered space $Y$ is attached to $\omega$ so that $\omega$ is a dense set of isolated points. If $\mathfrak{t}>\omega_{1}$ then $Y$ is densely linearly ordered and nowhere locally compact.

Making the whole space countably compact relies on the existence of numerous tight $\left(\omega_{1}, \mathfrak{c}^{*}\right)$-gaps and $\left(\mathfrak{c}, \omega_{1}^{*}\right)$-gaps. Some progress has been made in this direction - see the solution to Problem 10 of [11] in the Topology Atlas - but the models involved have "good" solutions to Problem 1 in them, and so Problem 3 remains open.

\section{Arbitrarily large first countable, locally compact, countably COMPACT SPACES}

In this section we turn to some related problems which may involve solutions to Problem 1. The following problem was featured in [9]:

Problem 5. Can there be an upper bound on the cardinalities of locally compact, first countable, countably compact spaces?

Without "locally compact" this would have an easy answer: take any regular cardinal and remove all its points of first countability. Problem 5 was originally motivated by Arhangelskii's famous solution to Alexandroff's old problem of whether there is an upper bound on the cardinalities of first countable, compact spaces. Arhangel'skii showed that $\mathfrak{c}$ is the upper bound. Both Problem 5 and Problem 6 below are generalizations of Alexandroff's problem. Problem 5 is also a generalization of the second part of Problem 1 in Juhász's article [5], which asks the same question (in negated form) of good spaces.

Problem 6. Can there be an upper bound on the cardinalities of locally compact, first countable, $\omega$-bounded spaces?

As far as we know, it may be consistent that $\mathfrak{c}$ is the upper bound in Problem 5 or Problem 6 as well; compare the first part of Problem 1 in Juhász's article. In any case, a positive solution even to Problem 6 would require the use of some very large cardinals.

Back when I first started thinking about Problem 5, I had not yet heard of the joint work of Juhász, Nagy and Weiss [6] which produced arbitrarily large splendid spaces, which are more than enough for a consistent No answer to Problem 5 . We now know that their construction works under e.g., Covering $(\mathrm{V}, \mathrm{K})$; for details see the article by Juhasz [5]. Thus it is consistent with $\mathfrak{c}$ being anything reasonable and requires large cardinals for its negation.

On the other hand, we also know [7] [5] that the Chang Conjecture variant $\left(\aleph_{\omega+1}, \aleph_{\omega}\right) \rightarrow\left(\aleph_{1}, \aleph_{0}\right)$ destroys all splendid spaces of size $\geq \aleph_{\omega}$. This variant, 
called the CCV below, has been shown consistent assuming a 2-huge cardinal. This use of the CCV has not been extended to a solution of Problem 6. The best we have so far is:

Theorem 3.1 ([7]). If the CCV holds, then every locally compact, locally hereditarily Lindelöf, $\omega$-bounded space is of Lindelöf degree $<\aleph_{\omega}$ and hence of cardinality $<\max \left\{\aleph_{\omega}, c^{+}\right\}$

For convenience I will temporarily adopt the following expressions:

Definition. A space is amenable if it is locally compact, locally hereditarily Lindelöf and countably compact and fine if it is locally compact, locally hereditarily Lindelöf and $\omega$-bounded.

Clearly, every splendid space is fine and every good space is amenable. A corollary of Theorem 3.1 is that the CCV implies every amenable space of Lindelöf degree $>\aleph_{\omega}$ contains a separable, countably compact noncompact subspace. This suggests that a ZFC construction of an amenable space of Lindelöf degree $\geq \aleph_{\omega+1}$ (in particular, a good space of cardinality $\geq \aleph_{\omega+1}$ ) would solve Problem 1. But it is conceivable that there may be one construction that works assuming the CCV, and another that works if the CCV fails, and which produces an $\omega$-bounded space in some models. This may not be the end of the story, however. Every amenable space that is not $\omega$-bounded contains a separable, noncompact closed subspace of cardinality $\leq \mathfrak{c}$. So if forcing is enough to destroy all such spaces, it seems plausible that a poset of modest size would be enough to do the trick. But the CCV is not destroyed by forcing by a set of cardinality lower than the first uncountable measurable cardinal. So it may not be a major step from the ZFC construction of an amenable space to an affirmative solution to Problem 1, or at least to a proof that large cardinals are needed to get a negative solution.

However, there is a far more sensational possibility: the hypothetical ZFC construction may actually be of a fine (perhaps even splendid) space, thereby showing that the CCV is inconsistent and hence so is the existence of 2-huge cardinals. Such a discovery would set off a major flurry of activity in large cardinal theory, as set theorists search for a natural lower bound for cardinals that are in jeopardy, so to speak.

On the other hand, the time may be ripe for lowering the large cardinal needed for the nonexistence of arbitrarily large fine (or at least splendid) spaces. A great deal has happened since [7] was published, including the discovery of Woodin cardinals and the equiconsistency of the Axiom of Determinacy (AD) with that of infinitely many Woodin cardinals. Recall that the consistency of AD was once thought to call for cardinals far larger than even 2-huge cardinals, and now it is known to call for something less than even a supercompact cardinal. If the consistency of nonexistence of arbitrarily large amenable spaces could be lowered this much, it would make their set-theoretic independence secure in the opinion of most set theorists.

A special case of fine, implicit in our next theorem [12], might give impetus to this quest. It uses the concept of a Kurepa family - a family $\mathcal{K}$ of denumerable 
sets which is uncluttered in the following way: for each countable $A \subset \cup \mathcal{K}$ the family $\mathcal{K}\lceil A=\{A \cap K: K \in \mathcal{K}\}$ is countable. A Kurepa family is called cofinal if it is $\subset$-cofinal in $[\bigcup \mathcal{K}]^{\omega}$.

Theorem 3.2. Let $\kappa$ be an infinite cardinal. The following are equivalent.

(a) There is a cofinal Kurepa family of cardinality $\kappa$.

(b) There is a locally metrizable, $\omega$-bounded 0 -dimensional space of weight $\kappa$.

The proof uses Stone Duality and the fact that a compact 0-dimensional space is metrizable iff it has at most countably many clopen sets.

Problem 7. Can 0-dimensionality be dropped from Theorem 3.2?

$1008 ?$

In Juhasz's article [5] the problem is posed whether (a) is equivalent to there being a splendid space of cardinality $\kappa$. Note that the spaces described in (b) are intermediate between splendid and fine spaces. The simple structure of cofinal Kurepa families suggests that a lowering of consistency strength as above may be within reach. The following problem may be especially tractable:

Problem 8. Is there a cofinal Kurepa family on $\mathbb{R}$ ?

\section{TOWARDS NEGATIVE ANSWERS}

In Section 1 we saw that there are good spaces that are not $\omega$-bounded if either $\mathfrak{t}=\omega_{1}$ or $\mathfrak{b}=\mathfrak{c}$. This, together with the Pigeonhole Principle applied to the well known fact [3] that $\mathfrak{t} \leq \mathfrak{b}$, implies that there are good spaces if either $\mathfrak{c}=\omega_{1}$ or $\mathfrak{c}=\omega_{2}$. This means that a negative answer to Problem 1 cannot be obtained by iterated forcing with countable supports: because this very popular and sophisticated method of producing models of set theory makes $\mathfrak{c} \leq \omega_{2}$. The main alternative, finite support forcing, can lead to models of $\mathfrak{c} \geq \omega_{3}$, but only if a tail of the iteration consists of ccc posets. One drawback is that ccc posets are a rather restrictive class. The technique of mixed supports does allow for some non-ccc posets while still leading to models of $\mathfrak{c} \geq \omega_{3}$, but we still have no good mixed-support candidates for negative answers to Problem 1. Another drawback of ccc forcing with finite supports is that Cohen reals are added at each limit stage, and these produce $\omega_{1}$-towers, all of which need to be destroyed if we want a counterexample to Problem 1. They can be destroyed without making $\mathfrak{b}=\mathfrak{c}$ or even $\mathfrak{b}=\mathfrak{d}$ : Section 7 of [11] gives one simple way, but with some choices of ground model the final model still has good spaces that are not $\omega$-bounded, and we still do not know whether all choices give good spaces.

On the other hand, models of $\mathfrak{c}=\omega_{2}$ have not been eliminated as candidates for negative answers to Problem 4, nor to the following problem:

Problem 9. Is there a scattered, countably compact $T_{3}$ space that can be continuously mapped onto $[0,1]$ ? 
While $\mathfrak{b}=\mathfrak{c}$ is enough to construct "good" examples for this problem [3, Theorem 13.4], no example at all has been constructed just assuming $\mathfrak{t}=\omega_{1}$ and we do not know whether $\mathfrak{c}=\omega_{2}$ is enough to construct one.

For countably compact Tychonoff spaces, admitting a continuous function onto $[0,1]$ is equivalent to having a non-scattered Stone-Čech compactification, and this is of interest in the geometry of Banach spaces of continuous functions [13]. Any example for Problem 9 contains a separable subspace that is also an example (just take the closure of any countable subspace whose image is the set of rational points in $[0,1])$. Since no compact scattered space can be mapped onto $[0,1]$, no example is $\omega$-bounded.

All the (consistent) examples for Problem 9 thus far constructed are first countable, in fact good, and I conjecture that Problem 9 is reducible to the locally countable, hence good case, making it a special case of Problem 1. A minimality argument involving the Cantor-Bendixson derivatives of a scattered space shows that if there is an example for Problem 9, it has a subspace $Y$ with the same properties in which each point has a nbhd which does not admit of a continuous map onto $[0,1]$. Such a nbhd meets at most countably many fibers $\pi^{-1}(r)$, since otherwise it would have a copy of the Cantor set in its image, and therefore it could be mapped onto $[0,1]$. If $Y$ has a point all of whose nbhds are uncountable [in other words, if $Y$ is not a good space] then every nbhd of that point must meet some fiber $\pi^{-1}(r)$ in an uncountable subset; moreover, $\pi^{-1}(r)$ is of Lindelöf degree $\mathfrak{c}$ because there are $\mathfrak{c}$ disjoint crowded countable subsets of $[0,1]$ with $r$ in their closure.

In contrast, it is very easy to construct a separable, locally countable, scattered, countably compact $\left(T_{2}\right)$ space that can be mapped continuously onto $[0,1]$, using the technique of [14] which adds points to compactify countable discrete subspaces and uses the resulting copies of $\omega+1$ to define a weak base for the topology. This highlights the importance of first countability in Problem 1 and of the $T_{3}$ separation axiom in Problem 9. It appears that the natural techniques for producing separable, locally countable, countably compact spaces either tie up the whole space into one compact (and countable) package, or else they tie up countable subsets as loosely as possible. The intermediate situation, where a fairly tight but noncompact tying-up is required, is where the challenging problems lie.

\section{REFERENCES}

[1] Alexandroff, P. and Urysohn, P., Mémoire sur les espaces topologiques compacts, Verh. Akad. Wetensch. Amsterdam 14 (1929).

[2] Aull, C., A note on countably paracompact spaces and metrization, Proc. Amer. Math. Soc. 16 (1965) 1316-1317.

[3] van Douwen, E.K., The integers and topology, in: Handbook of Set-Theoretic Topology, K. Kunen and J. Vaughan ed., North-Holland, 1984, pp. 111-167.

[4] Hechler, S., On some weakly compact spaces and their products, Gen. Top. Appl. 5 (1975) 83-93.

[5] Juhász, I., Good, splendid, and Jakovlev, this book. 
[6] Juhász, I., Nagy, Zs., and Weiss, W., On countably compact, locally countable spaces, Periodica Math. Hung. 10 (1979), 193-206.

[7] Juhász, I., Shelah, S., and Soukup, L., More on countably compact, locally countable spaces, Israel J. Math. 62 (1988), no. 3, 302-310.

[8] Nyikos, P. The theory of nonmetrizable manifolds, Handbook of Set-Theoretic Topology, K. Kunen and J. Vaughan ed., North-Holland (1984) 633-684.

[9] _ Progress on countably compact spaces in: General Topology and its Relations to Modern Analysis and Algebra VI, Proc. Sixth Prague Topological Symposium 1986, Z. Frolík ed., Berlin, Heldermann Verlag, 1988, pp. 379-410.

[10] _ On first countable, countably compact spaces II: Remainders in a van Douwen construction and P-ideals, Top. Appl. 35 (1990) 185-196.

[11] __ On first countable, countably compact spaces III: The problem of obtaining separable noncompact examples, Open Problems in Topology, J. van Mill and G. M. Reed ed., NorthHolland (1990) pp. 127-161.

[12] Generalized Kurepa and MAD families and topology, in preparation. Preliminary draft in http://www.math.sc.edu/ nyikos/preprints.html

[13] _ and Schäffer, J.J., Flat spaces of continuous functions, Studia Math. 42 (1972), $221-229$.

[14] _ and Vaughan, J., The Scarborough-Stone problem for Hausdorff spaces, Top. Appl. 44 (1992) 309-316.

[15] Vaughan, J., The Scarborough-Stone Problem, this book.

Department of Mathematics, LeConte College, 1523 Greene Street, University of South Carolina Columbia, SC 29208

E-mail address: nyikos@math.sc.edu 